\title{
Using Phase Shifts from High-order Harmonic Generated Radiation to Study Nuclear Dynamics
}

\author{
M. H. Mustary, D. E. Laban, J. B. O. Wood, I. V. Litvinyuk and R. T. Sang \\ Australian Attosecond Science Facility, Centre for Quantum Dynamics, \\ Griffith University, Brisbane, QLD 4111, AUSTRALIA \\ Corresponding Author: r.sang@griffith.edu.au
}

\begin{abstract}
We present initial phase shift measurements of high-order harmonic radiation generated from hydrogen isotopes using Gouy Phase interferometer. We will study the nuclear dynamics of molecules after they undergo strong-field ionization by ultrashort laser pulses.
\end{abstract}

\section{Introduction}

High harmonic generation (HHG) by an intense laser field has become one of the rapidly developing topics and well established method for the generation of coherent light in the extreme ultraviolet and soft $\mathrm{x}$-ray regions [1] as well as attosecond pulses [2]. HHG results when an electron is tunnel ionized from an atom or molecule, undergoes acceleration in the continuum, and then recombines with the parent ion. The amplitude and phase of HHG radiation can be studied as it contains information about the state of the atomic or molecular potential upon the electron's recombination. In investigating hydrogen, Baker et al experimentally showed that the heavier $\mathrm{D}_{2}$ isotope has higher HHG intensity compared to the lighter $\mathrm{H}_{2}$ isotope over a range of $\mathrm{HHG}$ energies [3]. From the ratio of the HHG intensity the dynamics of the nucleus were able to be studied on an attosecond timescale. However, there is still not sufficient work on observing phase shifts imparted onto HHG photons. In fact, detecting small phase shifts between the multiple sources of XUV light is difficult due to the very short wavelength and period. We investigate the phase shift in HHG of hydrogen isotopes i.e. hydrogen and deuterium using Gouy phase interferometer technique.

The Gouy phase interferometer technique [4] was developed in our laboratory and is able to measure the relative phase of two HHG sources when they are placed within a single laser focus. The phase shift in the interferometer originates from the Gouy phase of the driving laser as well as from the differences in atomic/molecular phases of the two sources. This technique is capable of observing the relative delay in electron recombination time between two argon gas jets with an accuracy of less than 100 zeptoseconds $\left(1 \mathrm{zs}=10^{-21} \mathrm{~s}\right)$ [5]. Thus, the Gouy phase interferometer is able to measure the very little phase shift between the hydrogen isotopes and can be useful to understand their nuclear dynamics.

\section{Gouy Phase Interferometer}

The Gouy phase shift describes the phase shift of the electric field of a focused laser as it propagates through the focus [6]. For a Gaussian beam the Gouy phase shift is defined as

$$
\phi_{\text {Gouy }}(z)=-\tan ^{-1} \frac{z}{z_{R}}
$$

Where $z_{R}$ is the Rayleigh length and $z$ is the distance from the beam waist within the focus along the axis of propagation. For a few-cycle laser pulse, the Gouy phase shifts the CEP along the propagation length and the behaviour predicted by Eq. 1 has been experimentally verified by Lindner et al [7]

The principle of operation of the Gouy phase interferometer is that two generation media are positioned within a single laser focus (gas jets at positions $\mathrm{z}_{1}$ and $\mathrm{z}_{2}$ ). The HHG emission depends on the phase of the fundamental generating laser which has been shifted between the two positions by the Gouy phase. The total phase shift in the HHG is related to the harmonic order $q$ and the Gouy phase is given by

$$
\phi_{H H G}=q\left[\phi_{\text {Gouy }}\left(z_{1}\right)-\phi_{\text {Gouy }}\left(z_{2}\right)\right]=q \Delta \phi \quad .
$$

Figure 1 shows the schematic of experimental set-up and the principle of operation of Gouy Phase interferometer. 


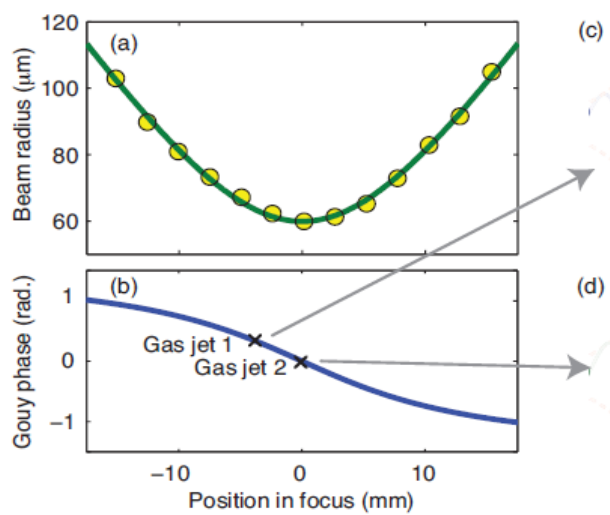

(c)

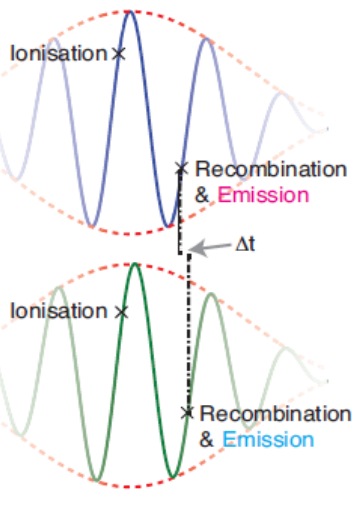

(e)

Two extreme ultraviolet pulses arrive at the detector with a phase offset

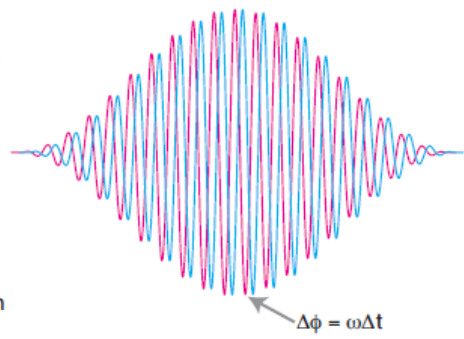

Fig 1: (a) the beam waist is measured at different positions along the focus (yellow circles) and a fit to these data points (green line) allows the Rayleigh range to be calculated. From the calculated Rayleigh range, the Gouy phase can be calculated at different focal positions (b). By situating two gas jets at different positions within the focus, the carrier-envelope phase of the driving laser gets shifted between interacting with gas jet 1 (c) and gas jet 2 (d). This results in a small delay time $\Delta t$ between the electron recombining. Finally, at the detector (e) two XUV pulses arrive with some phase offset either causing destructive or constructive interference to decrease or increase the total HHG yield respectively.

The chirped pulse amplification laser system in our laboratory produces $1 \mathrm{kHz}$ repetition rate $8 \mathrm{fs}$ pulses centered at $800 \mathrm{~nm}$ wavelength with average power of $500 \mathrm{~mW}$ and gives us the intensity approximately $1.0 \times 10^{15} \mathrm{~W} / \mathrm{cm}^{2}$ at the focus. This high intensity laser beam is passes through gas medium in a vacuum chamber where HHG is generated. The HHG photon then spectrally dispersed by a flat field XUV spectrometer, then intensified by MCP and the final image is captured by a CMOS camera.

\section{Investigating the phase shift in HHG between $\mathrm{H}_{2}$ and $\mathrm{D}_{2}$}

To investigate the HHG radiated phase produced in Gouy phase interferometer; two thin gas jets of $200 \mu \mathrm{m}$ in diameter are used as generation media with gas at a backing pressure of 100Torr. One jet is fixed in position whereas the other is translatable along the propagation of the laser beam. In our apparatus, each jet is connected with two fast solenoid valves that allow either $\mathrm{H}_{2}$ or $\mathrm{D}_{2}$ gas to be in the interaction region with the driving laser. Therefore allows us to check the interferometer in different configurations. The experiment protocol that we have developed requires that we vary the gas jet separation and collect three HHG spectra, two with either gas jet $\left(I_{l}, I_{2}\right)$ on and one with both gas jets on $\left(I_{12}\right)$. Once the intensities of the harmonics are extracted, the intensity is normalised to the coherent sum of the intensities generated by each gas jet to reveal an interference pattern. The normalized intensity, $I_{N}$, is expressed as

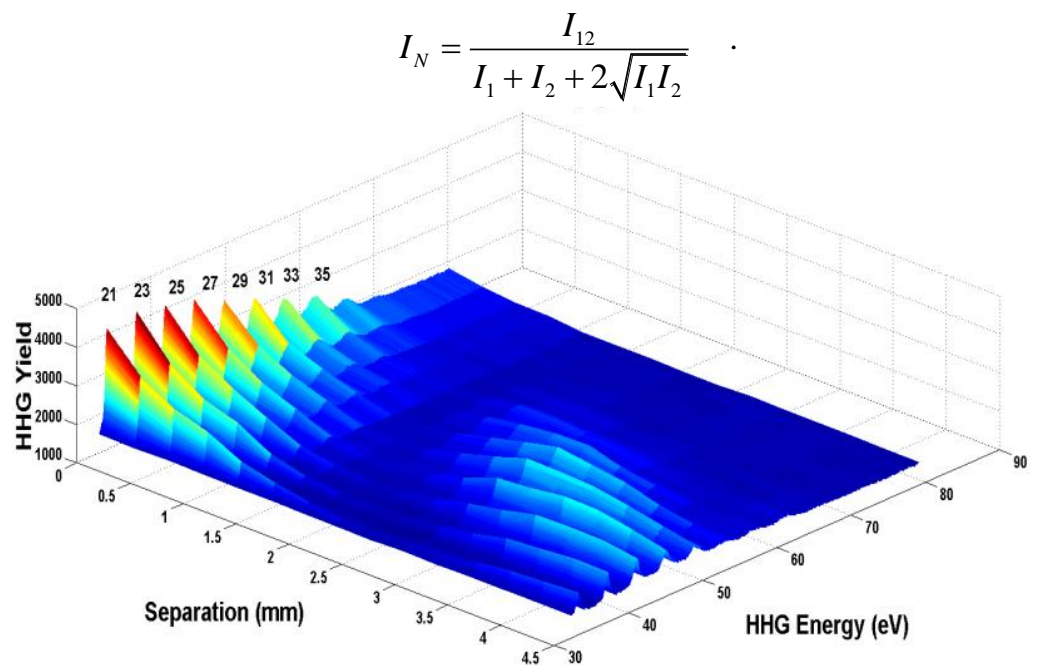

Fig 2: The observed temporal coherence of the harmonic orders 21 to 35 when the interferometer is configured so that both gas jets use $\mathrm{H}_{2}$ gas. This measurement confirms that the interferometer is working as expected.

Fig. 2 depicts the coherence of several harmonics when the separation between the gas jets is varied. This measurement is made to confirm the operation of the interferometer is working correctly using hydrogen in both gas jets. It can be clearly seen that each harmonic undergoes destructive and then constructive interference at a particular 
gas jet separation and increasing the harmonic number decreases the periodicity of constructive interference as it is predicted from Eq. (2). This is consistent with our previous measurements in [4].

In order to extract the relative phase between $\mathrm{H}_{2}$ and $\mathrm{D}_{2}$ we use two configurations of the gas jets. For the first set of measurement, we measure $I_{N I}$ with $\mathrm{H}_{2}$ gas is passed through one jet that is placed before the laser focus and $\mathrm{D}_{2}$ in the other jet that is placed at the centre of laser focus. Then the order of the gases is reversed and $I_{N 2}$ is measured. By subtracting the normalized HHG intensity of the second configuration from the first configuration and knowing the Gouy phase shift at the specific separation between the gas jets, the phase shift between $\mathrm{H}_{2}$ and $\mathrm{D}_{2}$ is then given by

$$
\Delta \phi=\frac{\Delta I_{N}}{\sin \left(q \phi_{\text {Gouy }}\right)} \text {. }
$$

Here, $\Delta I_{N}=I_{N 2}-I_{N 1}$ is the difference in the normalized intensity between the 2 sets of configurations as explained before. Gouy phase shift can be extracted from the temporal coherence observation by varying the separation between the gas jets when same gas species is passed through both jets $\left(\mathrm{H}_{2}\right.$ in this case) as shown in Fig. 2. The preliminary HHG phase shift for harmonic orders 21 to 33 is shown in Fig. 3. The separation between the gas jets is $0.635 \mathrm{~mm}$ and each data point is a statistical average of 200 spectra. We have observed $\sim 0.2-0.3 \mathrm{mrad}$ shift between $\mathrm{H}_{2}$ and D2 (red points). Reversing the gas order gives a negative phase shift but same magnitude (blue points) as expected.

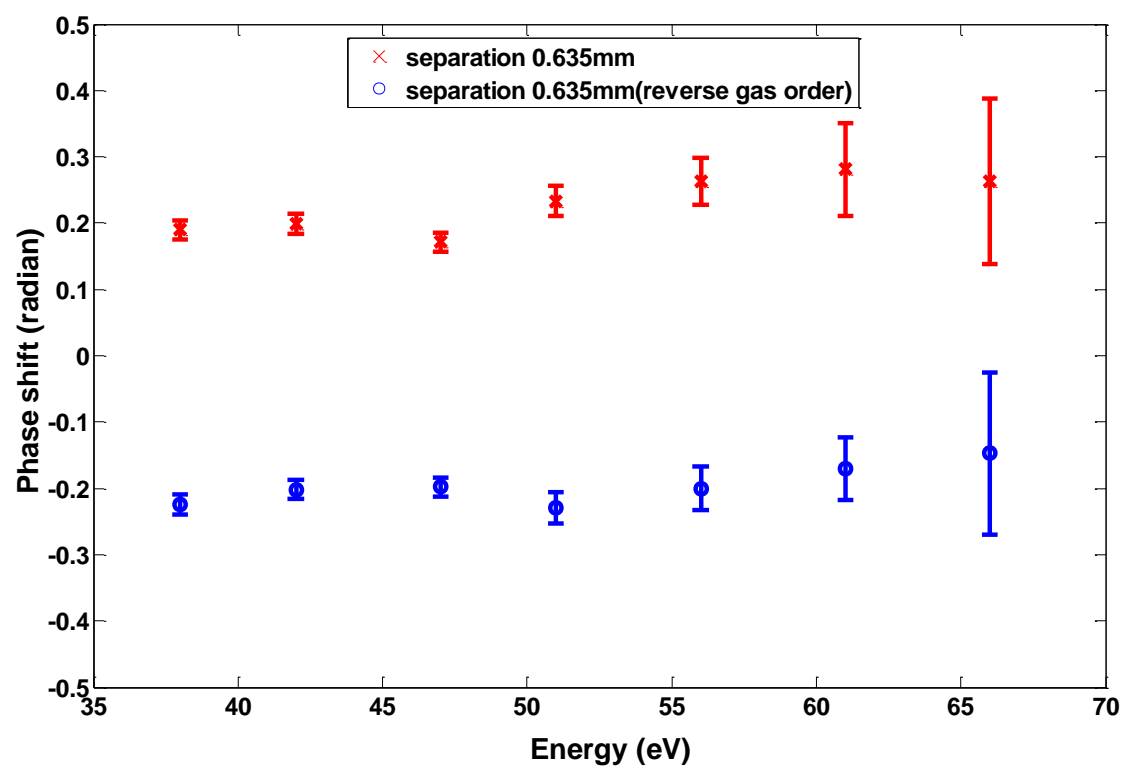

Fig 3: (a) HHG phase shift between $\mathrm{H}_{2}$ and $\mathrm{D}_{2}$ when the separation between the gas jets is $0.635 \mathrm{~mm}$. If positive phase (red points) is the phase of $\mathrm{H}_{2}$ relative to $\mathrm{D}_{2}$, then reverse the gas order (blue points) give the phase of $\mathrm{D}_{2}$ relative to $\mathrm{H}_{2}$

\section{Conclusion}

We are investigating the phase shift of HHG radiation as generated by hydrogen and deuterium using utilising the Gouy phase interferometer technique. Our preliminary data is consistent with our expectations and shows the great potential of this interferometer to study nuclear dynamics and structural arrangement in attosecond timescales.

This research was supported by Griffith University and the Australian Research Council.

\section{References}

[1] A. L'Huillier and Ph. Balcou, "High-order harmonic generation in rare gases with a 1-ps 1053-nm laser". Phys. Rev. Lett. 70, 774-777 (1993)

[2] F. Krausz and M. Ivanov, "Attosecond physics”, Rev. Mod. Phy. 81, 163 (2009).

[3] S. Baker, J. S. Robinson, C. A. Haworth, H. Teng, R. A. Smith, C. C. Chirila, M. Lein, J. W. G. Tisch, J. P. Marangos, "Probing Proton Dynamics in Molecules on an Attosecond Time Scale", Science 21, $312,424-427$ (2006).

[4] D. E. Laban, A. J. Palmer, W. C. Wallace, N. S. Gaffney, R. P. M. J. W. Notermans, T. T. J. Clevis, M. G. Pullen, D. Jiang,3 H. M. Quiney, I. V. Litvinyuk, D. Kielpinski, and R. T. Sang, "Extreme Ultraviolet Interferometer Using High-Order Harmonic Generation from Successive Sources", Phys. Rev. Lett. 109, 263902 (2012).

[5] D.E. Laban, W.C. Wallace, T.T.J. Clevis, N.S. Gaffney, M.G. Pullen, A.J. Palmer, D. Jiang, H.M. Quiney, I.V. Litvinyuk, D. Kielpinski, and R.T. Sang, "Zeptosecond timing resolution from a high-harmonic Gouy phase interferometer", Proceedings of the International Quantum Electronics Conference and Conference on Lasers and Electro-Optics Pacific Rim 2011.

[6] Gouy, L.G. Sur une propriete nouvelle des ondes lumineuses. C. R. Acad. Sci. Paris 110, 1251 (1890). 
[7] F. Lindner, G. G. Paulus, H. Walther, A. Baltuška, E. Goulielmakis, M. Lezius, and F. Krausz, "Gouy phase shift for few-cycle laser pulses", Phys. Rev. Lett. 92, 113001 (2004). 